\title{
Determinants of Department of Veterans Affairs hearing aid brand dispensing by individual audiologists
}

\author{
Earl E. Johnson, AuD, PhD \\ Mountain Home Department of Veterans Affairs Medical Center, Mountain Home, TN; Department of Communicative \\ Disorders, College of Clinical and Rehabilitation Health Sciences, East Tennessee State University, Johnson City, TN
}

\begin{abstract}
Department of Veterans Affairs (VA) audiologists were surveyed regarding their perceptions and evaluations of hearing aid (HA) brands on seven factors previously published in peer-reviewed research as important to the HA brand preference decision of audiologists. One of the seven factors formed a distinct dimension of brand differentiation based on Contract Pricing (dimension 1). Brand perceptions for the other six factors were highly correlated with one another; this dimension of correlated perceptions was labeled Propensity to Dispense Based on a Gestalt Percept (dimension 2). That is, a direct relation exists between the collective perception of HA brand and its likelihood of being dispensed. These two dimensions accounted for 93.1\% of the variance in the perceived differences among the HA brands surveyed. Joint-space mapping was used to model the effect of altering perceptions on VA HA brand dispensing. Results indicate that few VA audiologists (7.7\%) dispense HA brands in majority association with contract pricing. Instead, the vast majority (77\%) form brand preferences in majority association with their individualized perceptions.
\end{abstract}

Key words: audiologist, brand, contract, hearing aid, jointspace, mapping, perception, preference, pricing, VA.

\section{INTRODUCTION}

\section{Generalities of Purchasing Contracts}

Purchasing contracts are necessary or highly sought after when a buyer needs to acquire repetitively used items or products [1]. Establishing a purchasing contract has two major advantages. First, they are often an effi- cient mechanism for obtaining highly demanded products at a reasonable cost. Second, they limit the possible products for purchasing consideration to a reasonable number and help ensure quality and reliability of the products placed on the contract.

Purchasing contracts are not, however, without drawbacks. The most well-known drawback is a restriction on purchasing similar items not on contract when special needs arise. However, exceptions are sometimes made for extenuating circumstances or need. Another disadvantage is the cost of establishing a purchasing contract and implementing the process by which items are made eligible and evaluated for placement. The long-term savings of attaining items at a reasonable cost usually supersede the initial cost of contract establishment and implementation. In the end, the advantages of a purchasing contract often outweigh the disadvantages.

Purchasing contracts are common not only in businessto-business and business-to-client sales modalities within the private sector but also within government entities.

\footnotetext{
Abbreviations: DALC $=$ Denver Acquisitions and Logistics Center, FM = frequency modulation, $\mathrm{HA}=$ hearing aid, ROES = Remote Order Entry System, VA = Department of Veterans Affairs.

Address all correspondence to Earl E. Johnson, AuD, PhD; Mountain Home VA Medical Center, PO Box 4000, Audiology126, Mountain Home, TN 37684; 423-926-1171, ext 7149; fax: 423-979-3403. Email: earl.johnson@va.gov
}

DOI:10.1682/JRRD.2010.04.0077 
The private sector has long been regarded as the savvier implementer of the purchasing contract when compared with government entities [2]. Elemental drawbacks for government include the lack of centralization and integration and the necessity of guarding against unfair or corrupt use of powers by purchasing officials within government compared with private entities [2]. Nonetheless, purchasing contracts are the most ideal mechanism for meeting the daily operational needs of large government purchasing entities [2].

\section{Specific Federal Government Purchasing Contract in Audiology}

A long-time U.S. Federal Government purchasing contract in audiology involves the purchase of hearing aids (HAs) by the Department of Veterans Affairs (VA). For the first two quarters of 2009, the Hearing Industries Association reported that the VA dispensed 18 percent of all HAs sold in the United States. In addition to those HAs dispensed by the VA, the Federal purchasing contract is also used by other Federal entities, such as the Department of Defense, Indian Health Service, Health and Human Services, and Bureau of Prisons, for procuring HAs. The contract allows the purchase of commercially available HAs at an approximately 67 percent discount on manufacturer-suggested retail pricing. The contract currently operates on a 1 year cycle, with four 1 year renewal options, for the possibility of a 5 year cycle. The initial 1 year cycle involves a lengthy application and evaluation process ( 2.5 years) prior to and during consideration of HA brands and product options for purchase. At any one time, the VA contract for fiscal years 2004 to 2009 included 6 HA brands and approximately 100 HA product options. Product options are HA choices based on style, group, and technology level, all of which are specified in the purchasing contract. This article elaborates on a few details of these specifications. The latest award contract, effective from fiscal years 2010 to 2014, pending 1 year renewals, involves 9 HA brands and approximately 270 HA product options. The 5 year contract cycle's estimated value is $>\$ 1.5$ billion.

Thus, substantial financial interests are at stake for both the U.S. Federal Government and the HA industry. In general terms, the U.S. Government is interested in obtaining the most effective and efficacious HA products at a reasonable cost to taxpayers. Concomitantly, HA companies have a fiscal responsibility to stockholders to ensure that their own brand products are dispensed as often as possible at a fair return on investment. With an adequate return on investment, these companies can spend millions of dollars tracking trends in the HA marketplace. Examples of these expenditures are internal sales and marketing operations, as well as externally funding the Better Hearing Institute and the Hearing Industries Association. As a result, companies are often wellinformed on matters of the HA marketplace. The VA is also privy to much of this same information, with the exception of internal sales and marketing operation reports. In lieu of these internal reports, the VA has long operated its own internal tracking system of HA orders, the Remote Order Entry System (ROES). This system is operated by the Denver Acquisitions and Logistics Center (DALC) and provides an excellent source of data to study how audiologists dispense the various HA brands on the VA purchasing contract.

\section{Study Motivation and Rationale}

In recent years, surveys of audiologists have been undertaken to offer information pertaining to brand preference dispensing [3-4]. This area of research is regarded as important because a nationwide survey of audiologists in various work environments showed that 93.5 percent have a preferred brand that constitutes approximately 71 percent of their dispensed HAs [4]. A separate VA data analysis indicates that in 2009, more than three-quarters of VA audiologists dispensed more than 50 percent of their preferred HAs from one brand. The typical VA audiologist dispensed the majority of HAs from one brand 67 percent of the time [5]. An explanation for preferred HA brand dispensing has been previously pursued based on the generalized research of the Consumer's ValueAttitude System [6]. The Consumer's Value-Attitude System can be divided into the three components of an individual's belief system that have the potential to affect consumer purchasing: global values, domain-specific values, and evaluations of product attributes.

Global values are central to the identity of a consumer and include a set of both 18 instrumental and 18 terminal values [7]. For example, several instrumental values include ambitious (hardworking and aspiring), broad-minded (open-minded), capable (competent and effective), clean (neat and tidy), courageous (standing up for beliefs), forgiving (willing to pardon others), helpful (working for the welfare of others), and honest (sincere and truthful). Several examples of terminal values include a comfortable life (prosperous), equality (brotherhood 
and equal opportunity for all), an exciting life (stimulating, active), family security (taking care of loved ones), freedom (independence and free choice), health (physical and mental well-being), inner harmony (freedom from inner conflict), mature love (sexual and spiritual intimacy), and national security (protection from attack).

Domain-specific values are beliefs that apply to a specific circumstance or activity (e.g., HA brand dispensing). Evaluations of product attributes are beliefs about a particular class or category of products (e.g., HA brands). From the outset, the study of whether global values have an effect on HA brand dispensing was not theoretically supported because little rationale could be formed for why global values would affect the very domain-specific action of HA brand dispensing. Hence, research in this area began at the level of domain-specific values.

Johnson et al. studied 343 audiologists to examine whether personal values that are domain-specific to HA dispensing have an influence on brand preference choices [4]. A list of 32 items of potential importance to a brand preference decision was consolidated to seven statistically identified factors. The seven factors, in rank order of most to least importance, were (1) aptitude, (2) image, (3) cost, (4) sales and speed of delivery, (5) exposure, (6) colleague recommendation, and (7) contracts and incentives.

Johnson et al. identified that the aptitude factor consisted of the following principle components of a brand: reliability and quality of product, fit and/or comfort of product in patients' ears, customer service, and programming software [4]. The image factor consisted of innovativeness; research efforts; whether the distribution system is consistent with personal beliefs (e.g., not mass retail); design, features, and cosmetic appeal of product; and ability of product to match a prescriptive target for a patient's hearing loss. The cost factor included components of low price when compared with other brands and high value, i.e., it provides the most for its price. For clarification of principle components pertaining to the remaining factors, please refer to Johnson et al. [4].

Johnson et al. divided the 343 participating audiologists into groups based on their individualized preference for one of seven brands, with no less than 20 audiologists per group. Analyses determined whether the rated importance of those factors varied among the brand groups [4]. The mean importance of the factors, on a 0 (no importance) to 10 (highest importance) rating scale, did not differ for six of the seven most important factors. A few small but statistically significant differences did exist for the contracts and incentives factor, which was always rated as the least important. Thus, Johnson et al. concluded that these seven personal domain-specific values (statistical factors) did not differentiate the brand preference choices that audiologists were making.

The current study expands on the findings of Johnson et al. [4], with continued use of the Consumer's ValueAttitude System [6] as well as the Theory of Reasoned Action [8]. Evaluation of brand and/or product attributes that leads to the formation of beliefs or attitudes is likely to affect choices made by consumers (e.g., audiologists choosing HA brands). In other words, these choices are reasoned actions based on developed beliefs and attitudes, particularly given that domain-specific values have not been previously demonstrated to direct HA brand preference decisions. The personal evaluations (perceptions) that audiologists have of brand attributes, therefore, are hypothesized to differentiate individualized brand preference choices. If true, then by modeling the perceptual evaluations, we can better understand how audiologists arrive at the decision to dispense particular brands on a purchasing contract.

At the same time, these evaluations offer audiologists a mechanism through which to provide feedback about the HA brands on a purchasing contract. Such feedback from individuals regularly purchasing HAs under the purchasing contract is generally sought after. Because VA audiologists primarily dispense HA brands from the companies that are awarded contracts, the results in this article pertain to the use of brands on the contract. That is, a VA audiologist's brand preference, at least within the workplace, is constrained by the brands under contract; therefore, the perceptual evaluations and preferences of brands not available under a contract were not evaluated.

The purpose of this article is to identify the determinants of HA brand dispensing in the VA, many of which are thought to also affect brand dispensing by audiologists in the private sector. This study has not surveyed VA audiologists about the processes of patient care they use at the level of the individual patient. That is, audiologists often select treatment options for hearing loss based on patient-centered rehabilitative need, as well as pertinent contextual and environmental factors that relate to the patient in his or her day-to-day life. Hence, this study was not designed to evaluate such processes and pertains only to the narrow topic of HA brand selection and dispensing. 


\section{METHODS}

Two endeavors were undertaken to examine the perceptions audiologists have of HA brands on the VA purchasing contract. First, VA audiologists were surveyed to collect the perceptual evaluations of and preferences for the brands on contract. Second, actual HA orders made by VA audiologists were reviewed using the ROES to examine real brand dispensing patterns.

\section{Survey Questions}

Of all VA audiologists, 78 were surveyed between January 8, 2009, and February 13, 2009. Six of the audiologists voluntarily completed the survey twice to estimate test-retest reliability. The 78 audiologists represented 13 percent of the 600 audiologists who ordered at least 48 HAs during January and February 2009 based on ROES sales report data. The survey questions focused on five question sets.

The first question set included basic demographics and the audiologists' level of comfort, knowledge, and accuracy regarding their own assessment of HA brands. The survey collected these ratings on an 11-point rating scale ( -5 to 5 ), with 0 as a neutral point and the respective anchors of "very uncomfortable" to "very comfortable," "very unknowledgeable" to "very knowledgeable," and "very inaccurate" to "very accurate."

The second question set concerned perceptions of the seven factors previously demonstrated to be important to audiologists when they make HA brand preference decisions [4]. The survey obtained responses to these questions using another common response rating scale of 1 to 9, where 1 = worst and $9=$ best.

The third question set asked about audiologists' overall preference for the six HA brands on the VA purchasing contract on a rating scale of 1 to 9 , where $1=$ lowest preference and $9=$ highest preference. The survey placed the six HA brands on contract at the time of survey completion in alphabetical order: Interton (Ballerup, Denmark); MicroTech Hearing Technologies (Eden Prairie, Minnesota); Phonak AG (Stäfa, Switzerland); Siemens AG (Munich, Germany); Starkey Laboratories, Inc (Eden Prairie, Minnesota); and Unitron (Plymouth, Minnesota). Some of these brands are labeled by the same parent company and, in such cases, some similarities exist in product model functionality and programming software. However, because of the focus on brand perceptions, audiologists rated each separately.
The fourth question set asked audiologists to estimate the percentage of patients they fit with each brand on the purchasing contract. The survey collected responses within a range of 0 to 100 percent for each of the six brands, with the sum totaling 100 percent. These responses determined existing brand preferences.

The fifth question set asked audiologists to provide their perception of 17 common HA features, plus the HA programming software that they use (for a total of 18 items). Even though HA features and the programming software were statistical components of the aptitude and image factors, respectively (included in the second question set), the survey isolated these 18 items for perceptual assessment because of the concrete and often tangible nature of these HA aspects. The survey obtained responses to these questions using the common response rating scale of 1 to 9 , where $1=$ worst and $9=$ best. The survey specified the assessed items as follows: digital signal processing, feedback suppression, noise reduction, frequency modulation (FM) system compatibility, wireless technology (non-FM), automatic functionality (e.g., switching between listening programs), data-logging, acoustic telephone program, music program, adaptive directionality, volume control, integrated real-ear (i.e., at the time of survey completion, systems used real-ear-tocoupler difference to predict real-ear-to-aided response), telecoil, protection of devices from the elements (e.g., water, dirt, perspiration), wax guard devices, battery life, remote control operations, and programming software.

VA audiologists were notified of an institutional review board-approved online survey study of HA brands on U.S. Federal Government purchasing contract through a national Listserv requesting volunteer participation. Questions and response information were managed over the Internet with assisted survey hosting and maintenance through a commercial online survey development and management company (SurveyMonkey; Portland, Oregon).

\section{Data Analysis}

Simple descriptive statistics using SPSS version 14.0 (IBM; Somers, New York) analyzed the questions involving demographics and level of comfort, knowledge, and accuracy. Perceptual and joint-space mapping as employed by Marketing Engineering version 2.4 (DecisionPro, Inc; State College, Pennsylvania) completed the analysis of HA brand perceptions. Perceptual and/or joint-space mapping are prominent techniques for modeling brand attribute evaluations in the area of 
consumer behavior research [9]. These methodologies have been applied to not only brands, but also product designs, department stores, and even U.S. Presidential candidates [9]. In general, these maps identify specific strengths and weaknesses of the entities compared on multiple points of interest. Strategies for promoting strengths and improving weaknesses are then developed with the goal of later occupying a portion of the map that is most preferred by potential customers, which should in turn maximize market share and/or subsequent profits. Completing multiple maps over time can monitor changes in consumer perception (e.g., following storefront remodeling, a promotional event, or the introduction of new competition in the marketplace).

Mapping techniques, for either perceptual or jointspace maps, use factor analysis and multidimensional scaling statistical methods. To create maps of HA brands, VA audiologists were queried on a variety of attributes among competing brand alternatives. A rating scale of 1 to 9 was used, where 1 = the poorest assessment and $9=$ the best assessment, as has been recommended to perform assessments [9]. The data to extrapolate correlated attributes and form a smaller subset of orthogonal (independent) underlying factors that account for the maximum amount of variance among the surveyed attributes was used to complete a factor analysis. The data were then subjected to multidimensional scaling statistics. These statistics create maps, plotted in Euclidean space, that are graphical representations of perceived similarities or dissimilarities between a set of competing brands. For a more detailed explanation of perceptual map interpretation, refer to Lilien and Rangaswamy and Johnson [9-10].

Choice rules, using the brand preference ratings (e.g., rating scale of 1 [lowest preference] to 9 [highest preference]) as part of joint-space mapping, were also employed as an estimate of brand market share. As a separate estimate of brand market share, the percent of time each brand on contract was dispensed was calculated with a simple average of the reported percentages from participating audiologists. These market shares were estimates of the percentage of HAs dispensed across all brands on VA contract in early 2009.

Two common choice rules in joint-space mapping are first choice and share of preference. The first-choice rule assumes that each customer only purchases the most preferred product (the one closest to the ideal point or farthest along the preference vector). The share of preference rule assumes that each customer purchases every product in proportion to its measured preference value (relative to the sum of the preference values for all other brands in the model). The first-choice rule is appropriate for infrequently purchased products (e.g., cars), whereas the share of preference rule is appropriate for frequently purchased products (e.g., shampoos, soft drinks) [9]. The share of preference rule may be most appropriate for modeling HA market shares because VA audiologists frequently purchase or select HAs for patients.

\section{Review of VA Audiologist Hearing Aid Orders}

For direct comparisons of survey responses to actual data, the DALC ROES was used, because this system retains a record of HAs ordered by VA audiologists. To verify the accuracy of the subjective self-reported usage of HA brands by audiologist, the averaged response to question set 4 (percentage of HAs dispensed by brand) was compared with the actual market shares of the brands based on objective ROES data. Specifically, the DALC ROES application was used to retrieve facility-level audiology sales reports for the closest months of survey participation (i.e., January-February 2009). After deleting nonHA orders (e.g., remote controls, FM transmitters and receivers, and cochlear implants) included in the reports, a total of 63,692 orders remained. Comparisons of survey responses to actual data demonstrated that self-report data from audiologists, even including their perceptions, were largely accurate.

\section{RESULTS}

In the survey of 78 VA audiologists, 70.5 percent were female and 29.5 percent were male; this was reasonably consistent with the typical VA audiology employment sex of 76 percent female and 24 percent male (Veterans Health Administration Support Service Center human resource data report for January 2009). Reported work experience was $14.7 \pm 8.6$ years (mean \pm standard deviation). These 78 audiologists represented 17 of 23 different Veterans Integrated Service Networks (i.e., geographical divisions of the United States and its territories). Thus, these audiologists are assumed to be a representative sample of the larger population of VA audiologists.

Respondents perceived themselves as having considerable comfort, knowledge, and accuracy when it came to assessing the HA brands on the VA purchasing contract. On an 11 -point rating scale from -5 to 5 , where $-5=$ very 
uncomfortable and 5 = very comfortable, responses were significantly above the neutral point of 0 . Moreover, respondents reported more perceived comfort, knowledge, and accuracy when rating their own most preferred brand compared with all brands $(p<0.05)$. For the most preferred brand, responses averaged 3.2 across the areas of comfort, knowledge, and accuracy; for all brands, responses averaged 2.3 (Table 1).

The average audiologist ordered 18.3, standard deviation 10.5, HAs per week. Of the 78 participants, 82.9 percent had a brand they dispensed more than others. The average reported dispensing rate of that preferred brand was 63.9 percent, with no statistically significant difference among the six brands on contract (minimum = $61.0 \%$; maximum $=69.5 \%$ ). This average dispensing rate of the preferred brand was lower than reported elsewhere (i.e., $70 \%$ in the Johnson et al. study, which included many private practice audiologists who would be expected to benefit financially from a volume discount applicable when dispensing one brand predominantly [4]). A slightly lower dispensing rate of a preferred brand in a nonincentive dispensing environment such as the VA system is reasonable.

\section{Test-Retest Reliability of Brand Assessments}

Six audiologists repeated the perceptual assessments of all brands two weeks following the first survey. This provided an indicator of the test-retest reliability of brand assessments by audiologists. For the seven factors of varied importance to the HA brand preference decision of Johnson et al. [4], referred to as brand-centered factors, the six audiologists were very consistent on the repeated perceptual assessments $\left(F_{1,124}=561.474, r=0.905\right.$, $p \leq 0.001)$. With regard to the repeated preference assess-

Table 1.

Average ratings of how comfortable, knowledgeable, and accurate 78 Department of Veterans Affairs (VA) audiologists felt their assessment and preference ratings of hearing aid (HA) brands on VA purchasing contract were at time of survey completion. Note: 0 is neutral point of -5 to 5 rating scale.

\begin{tabular}{lccc}
\hline \multicolumn{1}{c}{ HA Brand } & Comfort $^{*}$ & Knowledge $^{\dagger}$ & Accuracy $^{\ddagger}$ \\
\hline Preferred Brand & 3.1 & 3.3 & 3.2 \\
All Brands & 2.1 & 2.4 & 2.4 \\
\hline${ }^{*}-5=$ very uncomfortable and 5 = very comfortable. \\
${ }^{\dagger}-5=$ very unknowledgeable and $5=$ very knowledgeable. \\
${ }^{\ddagger-5}=$ very inaccurate and 5 = very accurate. \\
\hline \hline
\end{tabular}

ments of the brands, the six audiologists were also very consistent $\left(F_{1,16}=74.169, r=0.907, p \leq 0.001\right)$. The constancy of these results is required of reliable measures used for validation of HA outcomes [11]. As examples, the Glasgow Hearing Aid Benefit Profile had an $r=0.86$ [12]; the Client Oriented Scale of Improvement had an $r=$ 0.84 [13]; and the Hearing Handicap Inventory in the Elderly, administered face-to-face, had an $r=0.96$ [14]. Although a repeated measure with more than six audiologists was attempted, only six repeated the 30 min survey because of apparent workload and time constraints.

\section{Perceptual and Preference Brand Assessments: Joint-Space Map}

Principle component factor analysis and multidimensional scaling statistics of the perceptual and preference assessments of the 78 audiologists indicate the presence of two dimensions underlying the seven factors (brandcentered attributes), as represented with a joint-space map (Figure 1). Vectors (blue lines) indicate the magnitude and direction of surveyed (brand) attributes in Euclidean space. Brands with higher ratings on an attribute vector are farther away from the origin of the graph on the same left or right hemisphere of the graph as the attribute label. Attributes at right angles to one another are considered uncorrelated, and attributes $180^{\circ}$ apart are considered negatively correlated. Attributes at angles of $45^{\circ}$ are considered to have a higher correlation as the degree of separation decreases, up to a maximum correlation of 1.0 for $0^{\circ}$ of separation. The main axes of the two-dimensional map, the ordinate and abscissa, are a special set of vectors that suggest the best underlying attributes or dimensions of how respondents differentiate brands. Perceptual maps can occur in more than two dimensions, but that was not the case in this study. Pink lines represent the direction of individual preferences for brands and attributes. The length of the both the blue and pink lines is based on the amount of variance accounted for with regard to each individual attribute and preference by the joint-space map. A longer line represents more preference for the attribute, whereas shorter lines represent less preference for the attribute [9]. A dimension was defined as having an eigenvalue of 1.0, which is a common cutoff value in multidimensional scaling statistics [15-16]. One eigenvalue equated to 14.3 percent of accounted variance (i.e., $100 \%$ divided by 7). The percentage of variance accounted for that is required for establishing a dimension is calculated by dividing the total variance (i.e., 100\%) by the number of items evaluated. In this study, seven items were evaluated; 


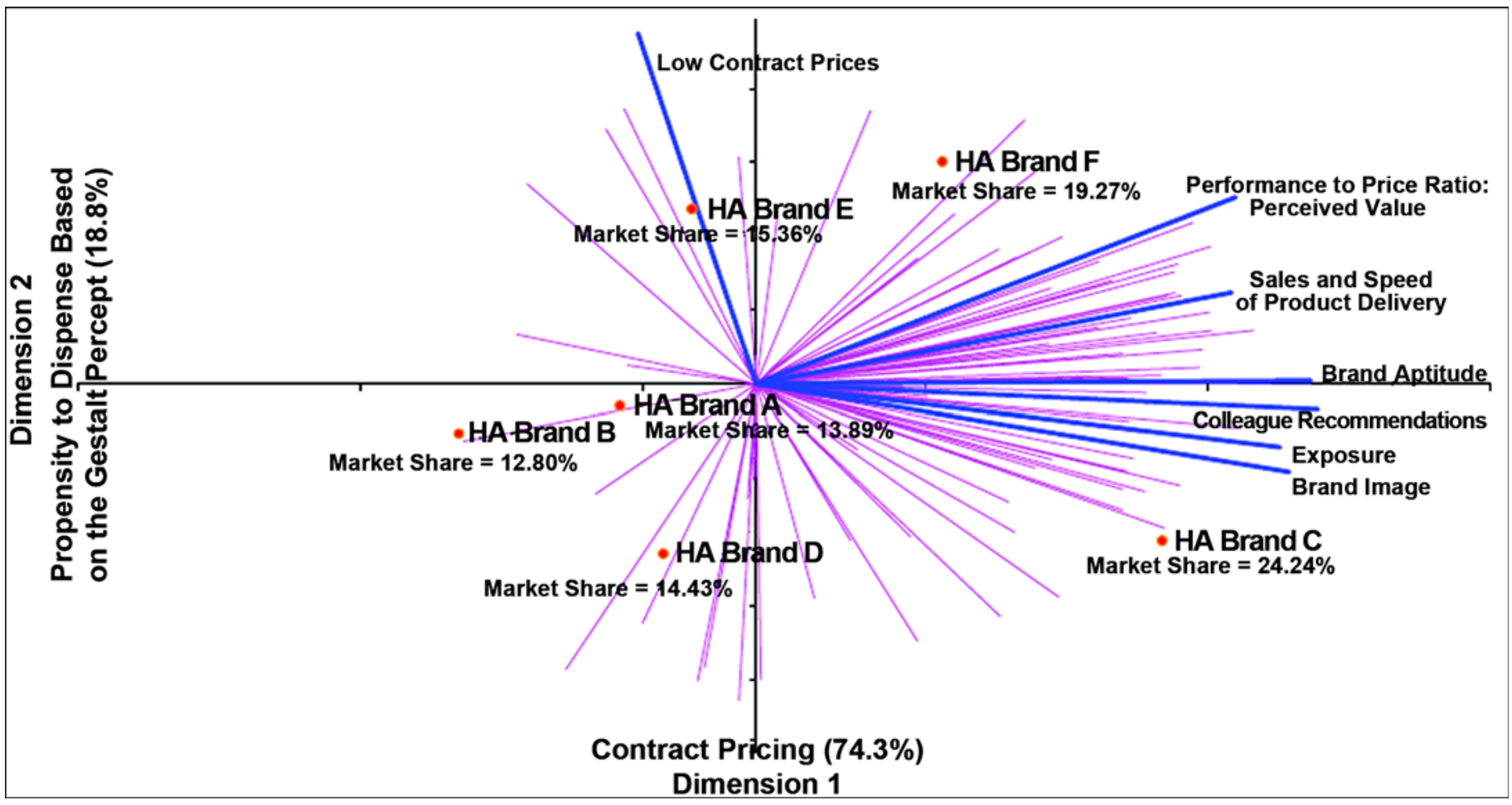

Figure 1.

Joint-space map of hearing aid (HA) brand attribute vectors (blue lines), preference vectors (pink lines), brand positions (orange circles), and brand market shares.

these items were taken from Johnson et al. [4]. For the seven brand-centered attributes, the average variance accounted for by the map for each attribute was 93.1 percent (range: $76.9 \%-99.3 \%$ for Sales and Speed of Product Delivery and Brand Aptitude, respectively). Considering the clustering of the factors around Contract Pricing (dimension 1) and Propensity to Dispense Based on Gestalt Percept (dimension 2), as well as estimated market share of the brands (based on preference data), the dimensions were labeled accordingly.

Dimension 1, representing 74.3 percent of the variance in the perceptual data, was labeled Contract Pricing because of the high correlation (0.86) between the factor Low Contract Prices and the ordinate axis in Figure 1. Dimension 2 was labeled Propensity to Dispense Based on the Gestalt Percept based on the fanning of factors around the abscissa axis, with an average interattribute correlation of 0.91 and the decline in market share percentages moving from positive to negative along the axis. Dimension 2 represented 18.8 percent of the variance in the perceptual data. The large correlations of each of the six factors with the abscissa were as follows: Brand Apti- tude $=0.997$, Colleague Recommendations $=0.97$, Exposure $=0.93$, Brand Image $=0.90$, Sales and Speed of Delivery $=0.88$, and Performance to Price Ratio: Perceived Value $=0.77$. These descending correlations then create a rank ordering of the association of each attribute with the Gestalt Percept. That is, Brand Aptitude, followed by Colleague Recommendations, and so on, is the ordering of perceptions that relate to the Gestalt Percept. The remaining factor of Low Contract Prices had a correlation of -0.21 with the abscissa because, as stated previously, this factor correlated most strongly with the ordinate axis $(r=0.86)$.

Figure 1 also shows, in addition to factors and dimension names, the positioning of brands in the perceptual space of VA audiologists. The singular position of each brand is represented by an orange circle. With regard to the preferences of individual audiologists, plotted as vectors (pink lines), the map accounted for 55.9 percent of the variance. Accompanying a randomized assigned letter to replace the actual brand name is a prediction of brand market share based on the reported preference data from the 78 audiologists using the share of preference choice rule. 


\section{Accuracy of Perceptions}

While it is impossible to quantify the accuracy of the perceptions held by the 78 audiologists for each of the seven brand-centered attributes (because no actual data exist for six of the attributes), objective data do exist for one attribute, Low Contract Prices. That is, actual pricing on the purchasing contract is always known; the question is whether audiologists are perceptually aware of differences in pricing across brands. The perceptual data along the attribute factor of Low Contract Prices were analyzed when the 78 audiologists grouped the six brands into three different price points (Figure 2). Likewise, analysis of actual contract pricing indicates a similar grouping of three price points, each separated by at least a 7.5 percent margin (DALC data). A more robust difference in average pricing of 15 percent was apparent when examining the behind-the-ear style only. Figure 2 clearly indicates that the $78 \mathrm{VA}$ audiologists were readily aware of these pricing differences with accurate perceptions.

\section{Predicted Versus Actual Brand Market Share}

Of relevance to the interpretation of market share data, an examination of the accuracy, or at least rank ordering, of the market share predictions with actual market share is important, because such validation lends support to the joint-space map itself. Additionally, the movement of brand position on the map is often used to indicate the effect of changing brand perception on brand market share.

Table 2 provides three columns of information. The first prediction, in column 1 , represents the reported percentages of how often HAs were dispensed by audiologists from the six brands on contract. For example, the average audiologist dispensed 35.7 percent of HAs from brand $\mathrm{C}$. The second prediction, in column 2, is based on a share of preference choice rule-the same prediction numbers shown in Figures 1 and 3. Column 3 represents the actual market share across brands as tallied from all HA orders in January and February 2009.

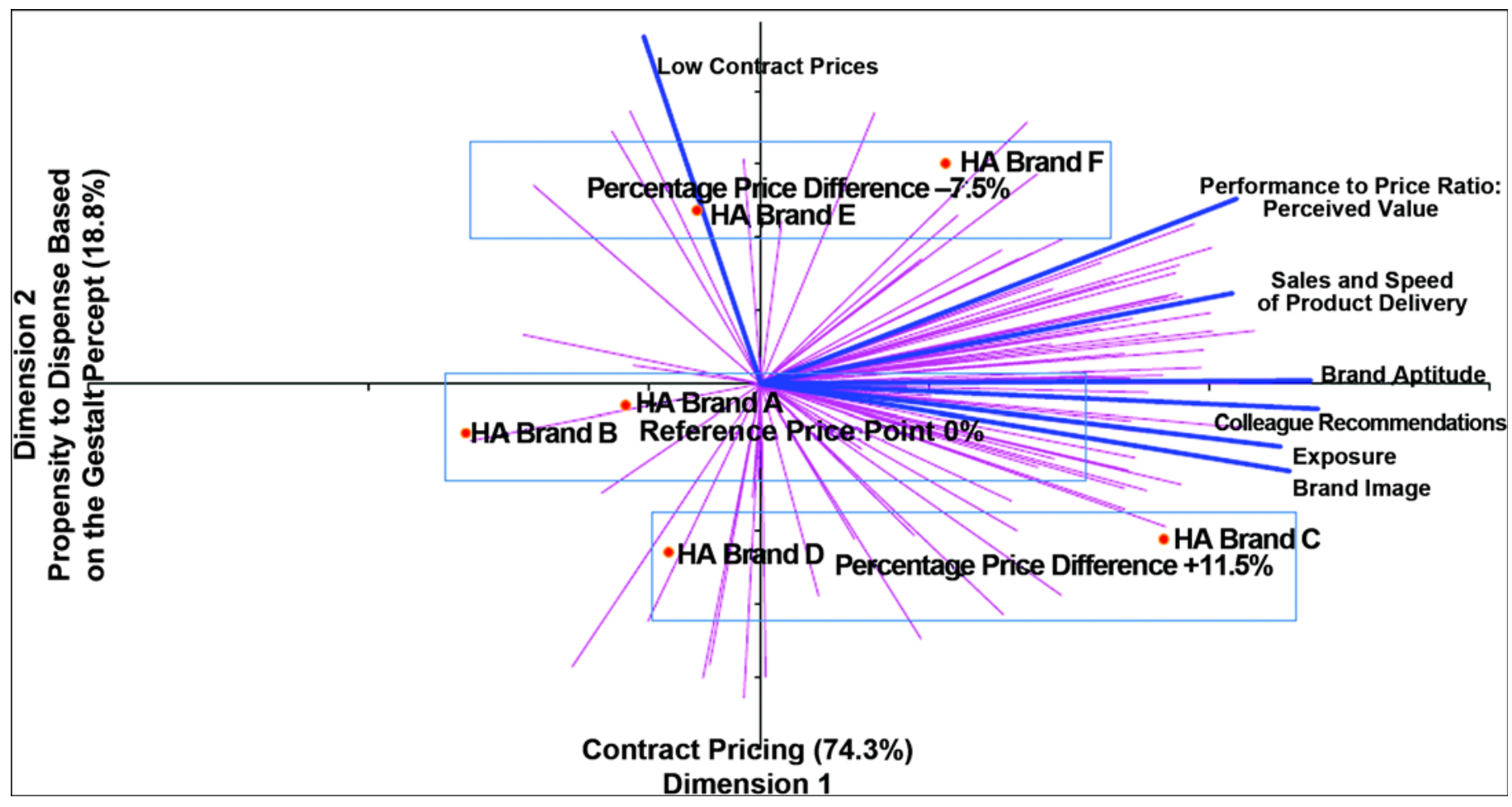

Figure 2.

Perception of pricing points by 78 audiologists is verified with averaged actual contract pricing data of behind-the-ear (BTE) and custom style hearing aids (HAs). Six HAs formed three pricing points separated by average percentage difference of 7.5 percent. More robust difference of 15 percent in average pricing was apparent when BTE style only was examined. 
Table 2.

Predicted and actual market share of hearing aid (HA) brands on Department of Veterans Affairs (VA) purchasing contract in January and February 2009. 1st prediction-reported dispensing rate percentage of HAs from six brands. 2nd prediction-share of preference choice rule. Actual-VA national sales summary data (January-February 2009) based on 63,692 individual HA orders.

\begin{tabular}{cccc}
\hline HA Brand & $\begin{array}{c}\text { 1st } \\
\text { Prediction (\%) }\end{array}$ & $\begin{array}{c}\text { 2nd } \\
\text { Prediction (\%) }\end{array}$ & Actual (\%) \\
\hline A & 8.6 & 13.9 & 6.3 \\
B & 11.5 & 12.8 & 5.3 \\
C & 35.7 & 24.2 & 36.8 \\
D & 10.5 & 14.4 & 11.0 \\
E & 12.4 & 15.4 & 15.1 \\
F & 21.7 & 19.3 & 25.5 \\
\hline \hline
\end{tabular}

The actual difference between columns 1 and 3 of Table 2 is quite small, a testament to the fact that audiologists have excellent knowledge regarding how often they dispense HAs of various brands. Differences between columns 2 and 3 are noticeably larger and indicate that the share of preference rule tends to overestimate market share for those with lower actual percentages and underestimate market share for those with higher actual percentages. Nevertheless, the rank ordering of market share by brand in Table $\mathbf{3}$ is the same among the actual data and the share of preference choice rule (i.e., column 2 predictions). Hence, the relative differences among brand market shares are maintained with the share of preference choice rule prediction utility. This consistency is necessary to allow the subsequent movement of brands in perceptual space to examine the effects of changing perception on predicted market share.

\section{Movement of Brands in Perceptual Space}

In Figure 3, the movement of brands $\mathrm{D}$ and $\mathrm{F}$ occurred vertically along the dimension of Contract Pricing (i.e., an upward movement modeled the effect of lowering contract price while a downward movement modeled the effect of raising contract price). Concomitantly, no horizontal movement occurred along the other brand attributes that contributed to a Gestalt Percept. Evidenced is the prediction that alteration in contract pricing has a miniscule effect on market shares of the two brands. The same was true for other brands as well; those brands were simply deleted from Figure $\mathbf{3}$ to aid in readability. Specifi-
Table 3.

Rank ordering of predicted and actual market shares, from highest (1) to lowest (6) for hearing aid (HA) brands on Department of Veterans Affairs (VA) purchasing contract in January and February 2009. 1st prediction - reported dispensing rate percentage of HAs from six brands. 2nd prediction - share of preference choice rule. Actual-VA national sales summary data (January-February 2009) based on 63,692 individual HA orders.

\begin{tabular}{cccc}
\hline HA Brand & $\begin{array}{c}\text { 1st } \\
\text { Prediction }\end{array}$ & $\begin{array}{c}\text { 2nd } \\
\text { Prediction }\end{array}$ & Actual \\
\hline A & 6 & 5 & 5 \\
B & 5 & 6 & 6 \\
C & 1 & 1 & 1 \\
D & 4 & 4 & 4 \\
E & 3 & 3 & 3 \\
F & 2 & 2 & 2 \\
\hline \hline
\end{tabular}

cally, the movement of brand $\mathrm{F}$ to a much higher contract price changed market share prediction by 0.01 percent, while the decrease in contract price for brand $\mathrm{D}$ eroded predicted market share by 0.23 percent.

\section{Explanation of Absolute Difference in Predicted Versus Actual Market Shares}

When using the share of preference choice rule, the anticipation was that predicted market shares would closely resemble actual market shares. As evidenced in Table 2, this was not the case. That is, the percentages in columns 3 and 4 were dissimilar. However, predicted market shares did follow the rank ordering of actual market shares (Table 3). The absolute difference between predicted and absolute market shares is because the preference vectors within the map only explain 55.9 percent of the variance in the preference data, while 44.1 percent of the preference data remained unexplained. A map explaining 55.9 percent of the variance in human behavior is considered, however, to be a good behavioral model. The overall $R$-value of the map model was $\sim 0.75$. Models with an overall $R$-value of $>0.5$ are considered of high magnitude when accounting for variation in human behavior [17].

Results of the mapping technique used in this study indicate that contract pricing has little to do with preference and, as a result, market share for the vast majority of audiologists (92.3\%). Of the 78 preference vectors (pink lines) in Figure 4, one for every survey respondent, only six (7.7\%) fall within $\pm 45^{\circ}$ (i.e., $r$-correlation of 0.5 or $R^{2}$ of 0.25 ) of the Low Contract Prices attribute. In contrast, $60(77 \%)$ of the 78 preference vectors in Figure 5 fall 


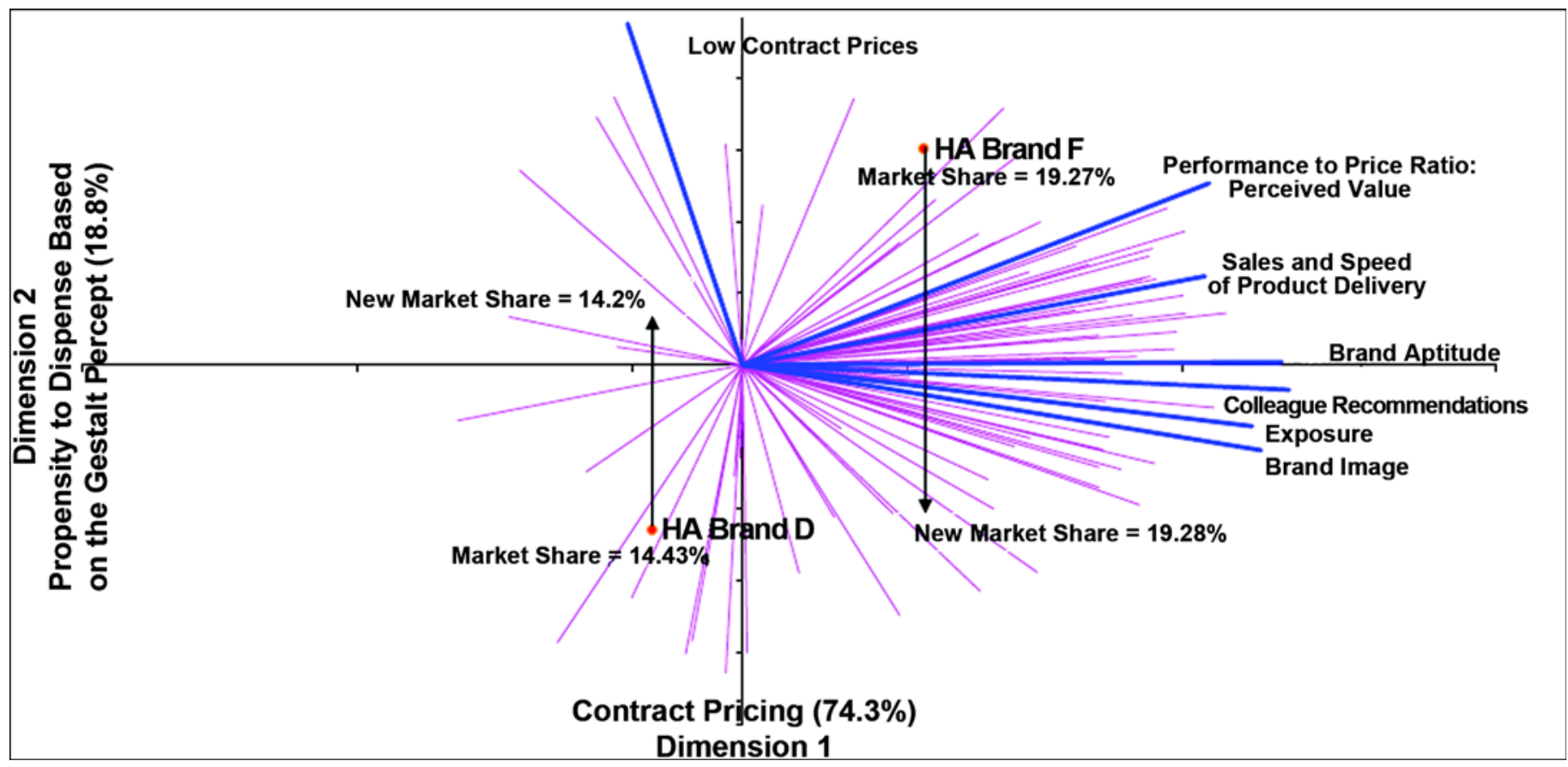

Figure 3.

Change in predicted hearing aid (HA) market share, based on changes in perception of contract pricing, indicating little to no effect of contract pricing on predicted market shares.

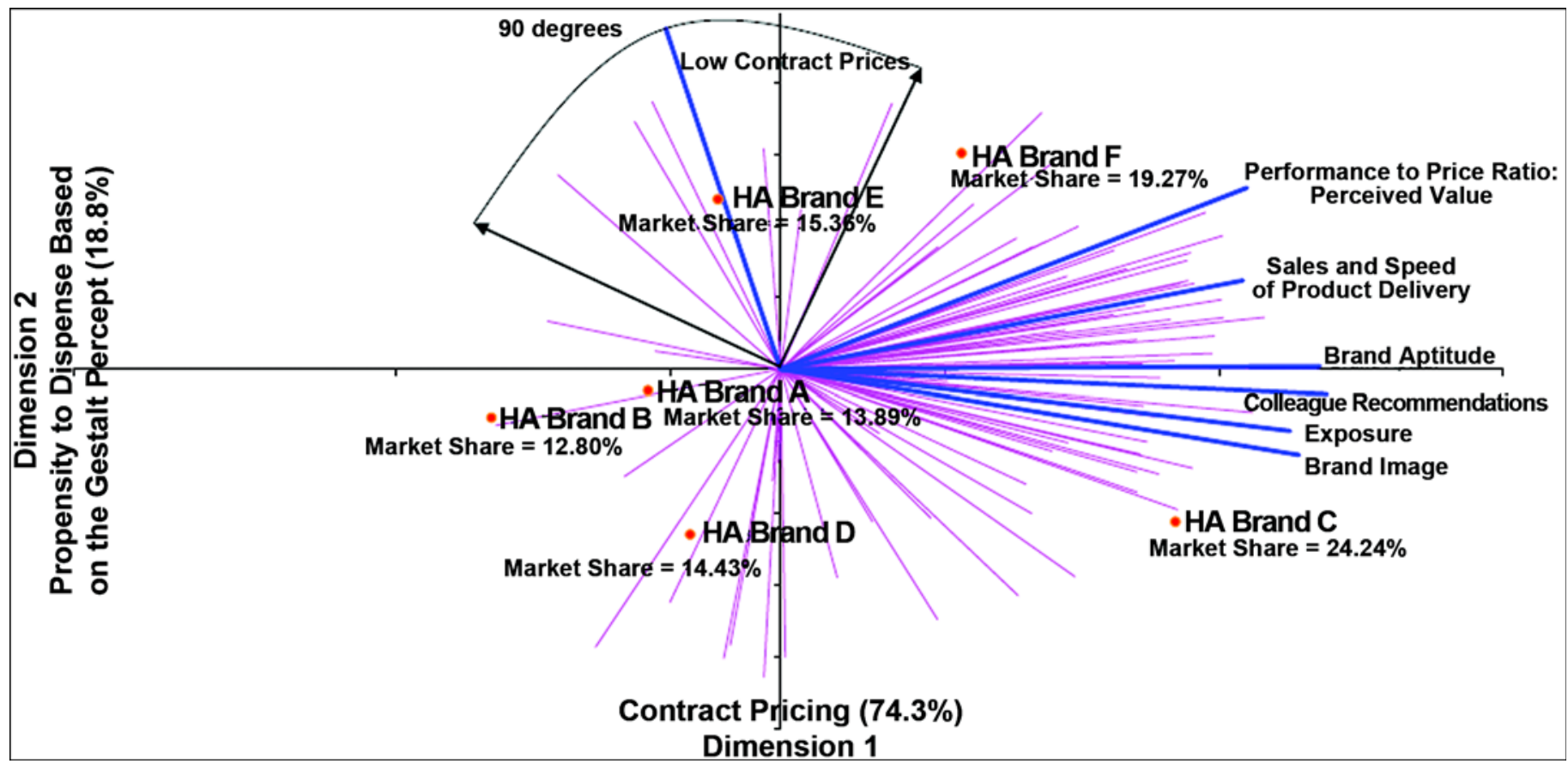

Figure 4.

Ninety degree arc around Low Contract Prices brand attribute (blue line) contains only six individual preference vectors (pink lines) (i.e., only $7.7 \%$ of all preferences). This is interpreted to mean that relatively few Department of Veterans Affairs audiologists formulate preference for HA brand based on low contract price. 


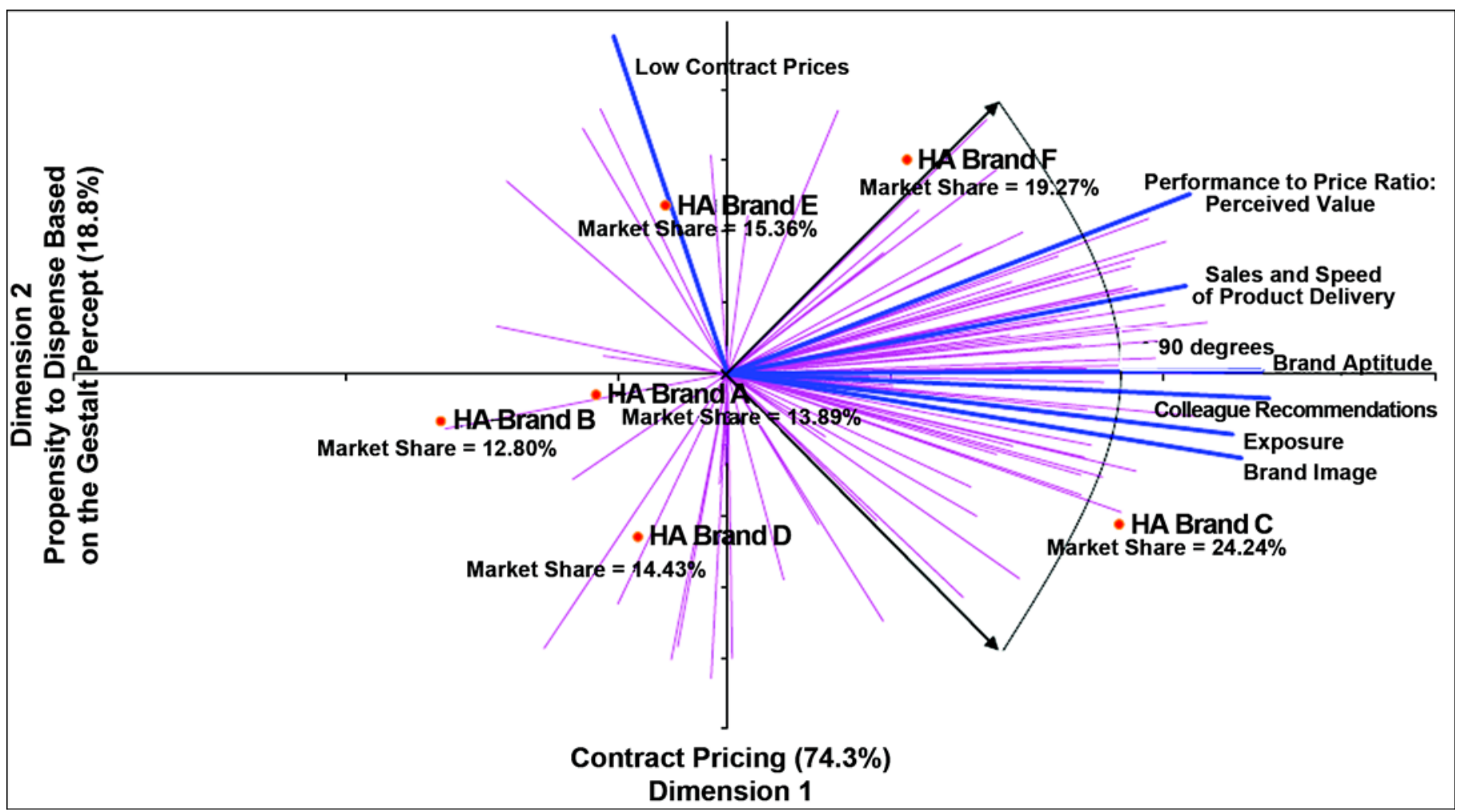

Figure 5.

Ninety degree arc around factor Propensity to Dispense Based on a Gestalt Percept (dimension 2), represented by abscissa axis, contains 60 individual hearing aid (HA) preference vectors (pink lines; i.e., 77\% of all preferences). This is interpreted to mean that over three-fourths of preferences that Department of Veterans Affairs audiologists have for HA brands are at least 25 percent explained by Gestalt Percept of HA brands.

within $\pm 45^{\circ}$ (i.e., $r$-correlation of at least 0.5 or $R^{2}$ of 0.25) of the Propensity to Dispense Based on a Gestalt Percept factor. This means that at least 25 percent of the preference decision is explained by the perceptual data for each of the 60 audiologists (77\%). Figure 6 also shows that $48(62 \%)$ of the 78 preference vectors fell within $\pm 22.5^{\circ}$ (i.e., $r$-correlation of at least 0.707 or $R^{2}$ of $0.50)$ of the Propensity to Dispense Based on a Gestalt Percept factor. In other words, for 48 (62\%) of the 78 surveyed audiologists, the brand preference decision is at least 50 percent explained by the Gestalt Percept.

\section{Perceptions of Product Features Across Brands}

Principle component factor analysis and multidimensional scaling statistics were also used in the creation of Figure 7. This map is based on the perceptions of the 78 VA audiologists of the 18 brand attributes shown, where $1=$ worst and $9=$ best. The 18 brand attributes were 17 product features and the programming software, as previously described in the "Methods" section, pertaining to question set 5 asked of the VA audiologists.

Using an eigenvalue cutoff of 1.0, with 5.5 percent of variance accounted for in this case, two dimensions were found to underlie these 18 brand attributes. These dimensions form the ordinate and abscissa axes of Figure 7. The axes were named based on the correlation of the attributes closest to each axis. All attributes, with the exception of Integrated Real-Ear and Remote Control, were more closely associated with the abscissa axis, i.e., all $R$-values $\geq 0.5$. The attribute of Integrated Real-Ear was most closely associated with the ordinate axis $\left(R=0.9, r^{2}=\right.$ $0.81)$. The attribute of Remote Control was more closely correlated with the ordinate axis $(R=0.61)$ but still had a significant correlation with the abscissa axis $(R=0.39)$; hence, the location of the attribute in the lower right-hand quadrant is somewhere between the abscissa and ordinate axes. In essence, these axes were labeled because of these patterns, similar to the method of labeling the axes in 


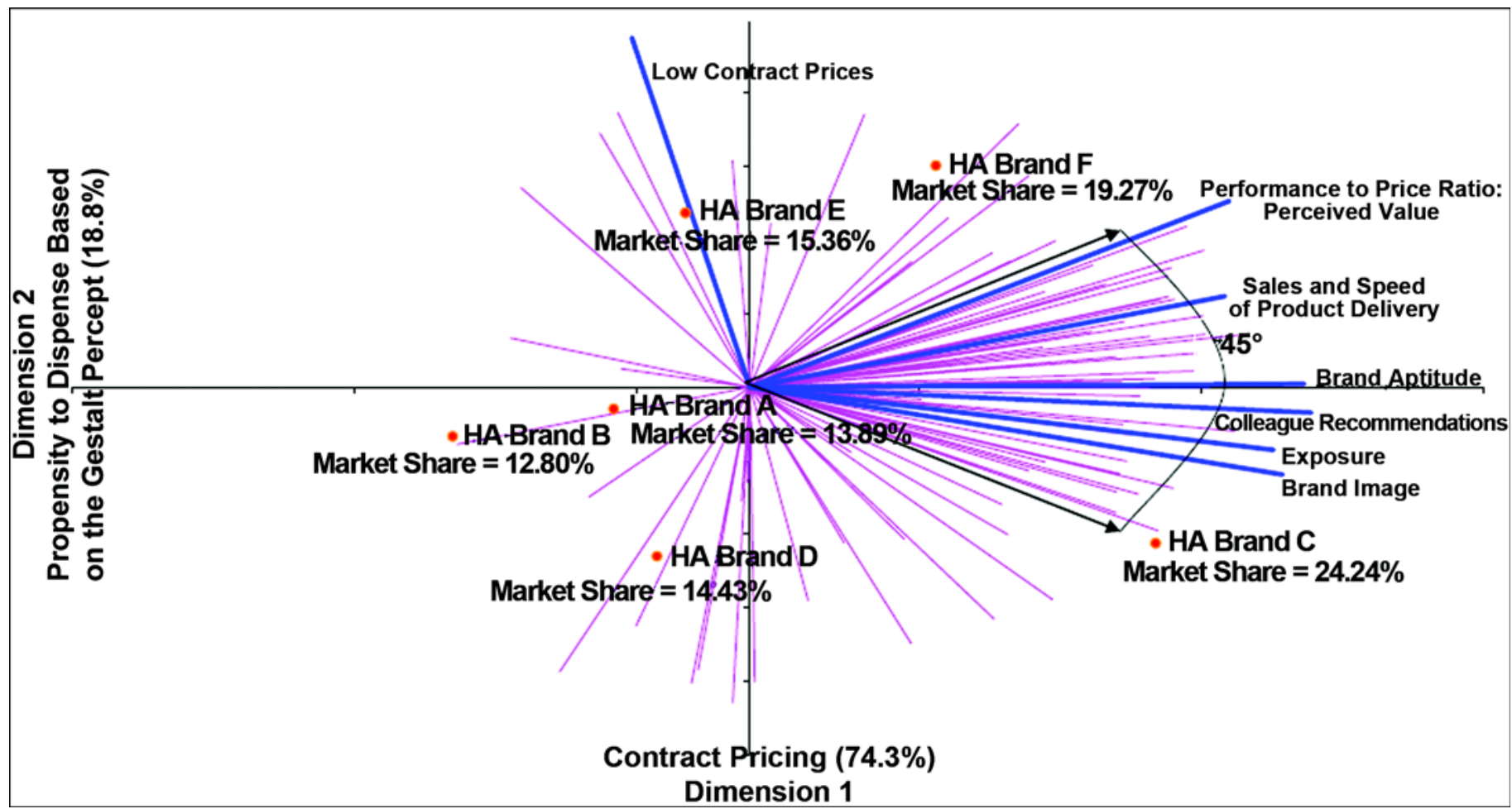

Figure 6.

Forty-five degree arc around factor Propensity to Dispense Based on a Gestalt Percept (dimension 2), represented by abscissa axis, contains 48 individual hearing aid (HA) preference vectors (pink lines; i.e., 62\% of all preferences). This is interpreted to mean that for these audiologists, their HA preferences are at least 50 percent explained by Gestalt Percept of HA brands.

Figures 1 to 6. The abscissa axis was labeled Propensity to Dispense Based on a Gestalt Percept, because predicted market shares (not shown for readability) decreased from right to left. The abscissa axis was labeled Integrated Real-Ear versus No Integrated RealEar, not only because of the perceptual map but also because brands $\mathrm{E}$ and $\mathrm{F}$ actually had the feature, whereas brands A, B, C, and D did not. Collectively, the two dimensions explained 97 percent of the variance in perceptions across the six brands on contract.

Despite dimension labeling, the more intriguing result illustrated with this map is how the brands compared with one another on the 18 attributes. Each attribute was not perfectly associated with the abscissa or ordinate axis, and this map illustrates this with the attribute vectors (solid blue lines). Traversing each attribute vector, from the point most distal from the origin to the origin and beyond into the opposite quadrant (added with a dashed blue line in Figure 8), we can achieve a rank ordering of the brands along the attributes. That is, by drawing vertical lines from the Noise Reduc- tion attribute vector (blue line) to intersect with the brand labels (orange circles, Figure 8), we can see that brand C is perceived as best (most distal from the origin on the solid blue vector), followed by brands F, A, E, D, and B for this attribute (A and $\mathrm{E}$ were essentially tied). Likewise, for the Adaptive Directionality attribute, we can see that brand $\mathrm{C}$ is perceived as best, followed by brands $\mathrm{F}$, A, D, E, and B (A and D were very close to one another perceptually). For the Integrated Real-Ear attribute, brand $\mathrm{F}$ is perceived as best, followed by brand $\mathrm{E}$. Brands $\mathrm{A}, \mathrm{B}, \mathrm{C}$, and D were known to not have the Integrated Real-Ear feature. Thus, these brands are plotted below the abscissa with no extension of the Integrated Real-Ear attribute (dashed blue line into its opposite quadrant).

Simply stated, Figure 7 is a straightforward map indicating the perceptions of features across brands. Because of the Gestalt Percept brand $\mathrm{C}$ was typically perceived as the best, with the exception of the Integrated Real-Ear attribute. Moreover, the differences between brands A, B, D, and E were generally minimal. Table 4 shows mean data for the brand attributes across brands. 


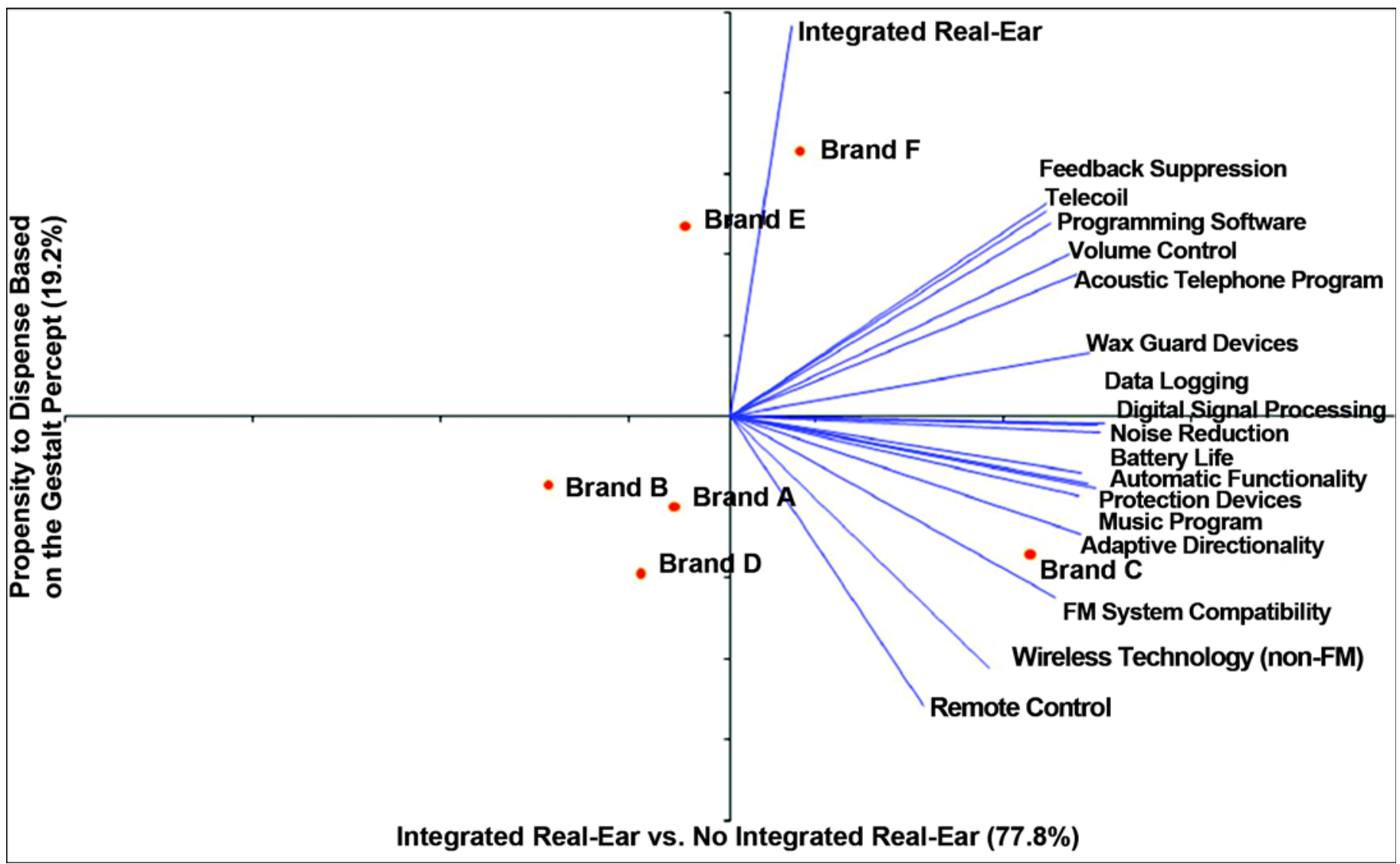

Figure 7.

Perceptual map of hearing aid brands positioned based on product feature ratings of 78 Department of Veterans Affairs audiologists. FM = frequency modulation.

In fact, the same data shown in Table 4 were used to generate a statistical map in Figure 7. The perceptual data agree well with the actual brand purchasing in January and February 2009, as shown in Table 2. Brand C had a 36.8 percent market share and brand $\mathrm{F}$ had a 25.5 percent market share; these were accordingly perceived as first and second best overall with regard to the 18 brand attributes assessed (Table 4).

\section{DISCUSSION}

A major goal of the VA National Hearing Aid Program is to provide the best, most current HA technology to veterans at the best price on purchasing contract. By ensuring that only top-quality HA products are available, the contract allows audiologists to address the auditory rehabilitation of veterans with hearing impairment while considering pertinent contextual and environmental factors rather than focusing on the product. The importance of product cost as a determinant of brand choice is also lessened, because brand offerings are priced in a fairly comparable manner. An advantage of currently (2011) having nine brands and a large number of product models on the purchasing contract (277 at the time of data collection for this study) is that the individual audiologist can find his or her favorites and, as a result, are presumably content with the HAs he or she chooses to dispense.

The multidimensional scaling analysis identified two dimensions underlying HA brand choices on the VA purchasing contract; however, the dimension of Contract Pricing was shown to have little effect, if any, on predicted market shares. This finding was in spite of the fact that VA audiologists were acutely and accurately aware of minimal contract pricing differences across brands. Instead, the results indicate that other perceptions of HA 


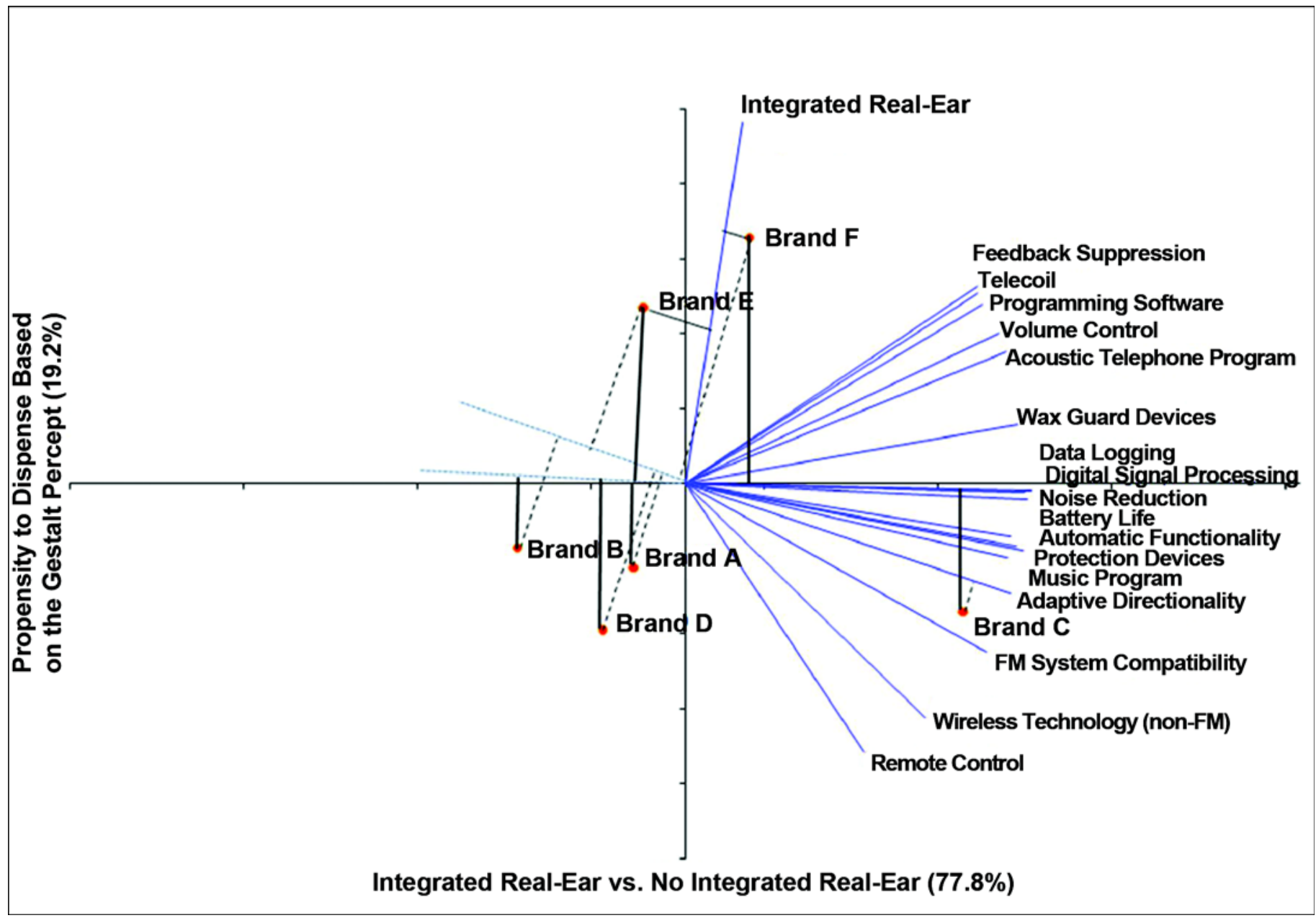

Figure 8.

Demonstration of how rank orderings of hearing aid (HA) brands, from best to worst as perceived by 78 Department of Veterans Affairs audiologists, can be achieved for each attribute using statistical mapping. Each attribute is represented by a vector (solid blue line). Vectors are extended into adjacent quadrants (dashed blue lines) for those attributes demonstrated in this figure. Solid thin black line connects with integrated real-ear vector. Note that HA brands A through D do not have solid thin black line connecting to integrated real-ear vector because these brands did not have this feature available. Solid thick black line connects with noise reduction vector. Dashed black line connects with adaptive directionality vector. Note that all HA brands connect to noise reduction and adaptive directionality vectors because each brand had these features available in commercial HA products. FM = frequency modulation.

brand attributes are the driving force of brand choices (market share percentages) with a better Gestalt Percept leading to higher market shares and vice versa. That is, the more highly regarded a brand is by the audiologist, the more likely it will be dispensed. A positively regarded outcome of the study is that audiologists also felt comfortable, knowledgeable, and confident in the accuracy of their perceptions.

The effect of a better Gestalt Percept on brand market share is evidenced by the vast majority of individual preference vectors (pink lines) that assemble in the top and bottom quadrant regions to the right of the ordinate axis in Figure 5. Likewise, the lack of a significant effect on market share by adjustment to contract pricing is shown in Figure 3. This data regarding the lack of an effect of pricing on brand dispensing does have limited applicability within the VA model and likely does not extend into a private sector model. That is, the remuneration of VA audiologists is independent of the product profit margin, whereas price of the product does affect profitability and sustainability of a private practice and, in many cases, the remuneration of a privately employed audiologist. It would 
Table 4.

Mean rating of attributes across hearing aid (HA) brands provided by 78 Department of Veterans Affairs audiologists. Overall average rating provides ranking of HA brands as perceived by audiologists regarding survey features. In addition, HA brands can be ranked for any specific feature.

\begin{tabular}{|c|c|c|c|c|c|c|}
\hline Attribute & Brand A & Brand B & Brand C & Brand D & Brand E & Brand F \\
\hline Digital Signal Processing & 6.3 & 5.7 & 8.1 & 6.2 & 6.3 & 6.9 \\
\hline Feedback Suppression & 6.0 & 5.3 & 7.5 & 5.4 & 6.8 & 7.5 \\
\hline Noise Reduction & 6.1 & 5.5 & 7.6 & 5.8 & 6.0 & 6.5 \\
\hline Automatic Functionality & 5.9 & 4.9 & 7.8 & 5.7 & 5.6 & 6.1 \\
\hline Data Logging & 5.9 & 5.5 & 7.7 & 6.1 & 6.2 & 6.6 \\
\hline Programming Software & 5.2 & 4.9 & 6.3 & 5.2 & 5.9 & 6.3 \\
\hline Adaptive Directionality & 6.1 & 5.7 & 7.7 & 6.0 & 5.7 & 6.3 \\
\hline FM System & 3.4 & 2.4 & 8.3 & 3.4 & 2.6 & 2.8 \\
\hline Volume Control & 6.0 & 5.5 & 7.0 & 5.8 & 6.4 & 6.8 \\
\hline Integrated Real-Ear & 2.1 & 1.7 & 2.3 & 1.9 & 5.5 & 6.8 \\
\hline Remote Control & 4.9 & 1.9 & 7.2 & 5.9 & 1.8 & 2.0 \\
\hline Average Rating & 5.3 & 4.7 & 6.9 & 5.3 & 5.3 & 5.8 \\
\hline Overall Ranking & 3rd & 6th & $1 \mathrm{st}$ & 3rd & 3rd & 2nd \\
\hline
\end{tabular}

therefore seem that pricing would play a larger role on brand dispensing in the private sector than within the VA.

\section{CONCLUSIONS}

Positive evaluations and perceptions (attitudes) of HA brand attributes had a highly significant association with the selection of a preferred HA brand for 77 percent of the VA audiologists surveyed. This finding was consistent with the Consumer's Value-Attitude System [6], which indicates that attitudes will likely determine consumer choice, given that values had previously been demonstrated to not differentiate HA brand preference decisions [4]. Only a few VA audiologists (7.7\%) selected a preferred HA brand in majority association with low contract pricing. HA brand attribute perceptions are based largely on the unique experiences of individual audiologists with the brands rather than large-scale evidence of brand offering comparisons (because these do not exist). Despite the lack of a contract pricing effect on brand dispensing, establishing reasonable, homogenous contract prices for the procurement of HAs is expected to continue. Reasonable pricing provides an excellent means of limiting the overall expenditures of the VA National Hearing Aid Program. Additionally, the use of perceptual and joint-space mapping was demonstrated as one technique that can be used for modeling the dispensing perceptions and preferences of VA audiologists. This modeling can be used to not only study HA brand preference dispensing but also portray the perceptions of a large number of audiologists concisely. Audiologists may derive value from the collective perceptions and preferences of their peers in the absence of scientific evaluations regarding HA brands and their product offerings. This, of course, presumes that these perceptions and preferences prove true. One hopes so; after all, these perceptions are how HA brand dispensing decisions are made by the individual audiologist.

\section{ACKNOWLEDGMENTS}

Financial Disclosures: The author has declared that no competing interests exist.

Funding/Support: This material was based on work supported by the Mountain Home VA Medical Center Auditory and Vestibular

Research Enhancement Award Program and a career development award sponsored by the VA Rehabilitation Research and Development Office. 
Additional Contributions: The author would like to thank Drs. Todd Ricketts, Richard Wilson, and Colleen Noe for their comments and suggestions during manuscript preparation.

Institutional Review: This survey was approved by the East Tennessee State University/Mountain Home VA Medical Center institutional review board.

Participant Follow-Up. The author plans to inform participants of the publication of this study.

Disclaimer: The opinions expressed in this article are those of the author and do not necessarily represent the official position of the VA or the U.S. Government.

\section{REFERENCES}

1. Noordewier TG, John G, Niven JR. Performance outcomes of purchasing arrangements in industrial buyer-vendor relationships. J Marketing. 1990;54(4):80-93. DOI:10.2307/1251761

2. Thomas AG. Principles of government purchasing. New York (NY): BiblioLife; 2009.

3. Johnson EE. Survey explores how dispensers use and choose their preferred hearing aid brands. Hearing. 2007;60(3): 23-36.

4. Johnson EE, Mueller HG, Ricketts TA. Statistically derived factors of varied importance to audiologists when making a hearing aid brand preference decision. J Am Acad Audiol. 2009;20(1):40-48. [PMID: 19927681]

DOI:10.3766/jaaa.20.1.4

5. Johnson EE. How do hearing aid dispensers pick their buying preferences? Hearing. 2010;63(3):10-14.

6. Vinson DE, Scott JE, Lamont LM. The role of personal values in marketing and consumer behavior. J Marketing. 1977;41(2):44-50. DOI:10.2307/1250633

7. Rokeach MJ. Beliefs, attitudes, and values. San Francisco (CA): Jossey Bass; 1968.

8. Fishbein M, Ajzen I. Belief, attitude, intention, and behavior: An introduction to theory and research. Reading (MA): Addison-Wesley; 1975.

9. Lilien GL, Rangaswamy A. Marketing engineering. 2nd ed. Upper Saddle River (NJ): Prentice Hall; 2003.
10. Johnson EE. What's important to audiologists and their individual decision choice? [Internet]. San Antonio (TX): Audiology Online; 2008. Available from: http://www .audiologyonline.com/articles/article detail.asp?article id=2140

11. Hyde ML. Reasonable psychometric standards for selfreport outcome measures in audiological rehabilitation. Ear Hear. 2000;21(4):24S-36S. [PMID: 10981592] DOI:10.1097/00003446-200008001-00005

12. Gatehouse S. Glasgow Hearing Aid Benefit Profile: Derivation and validation of a client-centered outcome measure for hearing aid services. J Am Acad Audiol. 1999;10:80-103.

13. Dillon H, Birtles G, Lovegrove R. Measuring the outcomes of a national rehabilitation program: Normative data for the Client Oriented Scale of Improvement (COSI) and the Hearing Aid User's Questionnaire (HAUQ). J Am Acad Audiol. 1999;10:67-79.

14. Weinstein BE, Spitzer JB, Ventry IM. Test-retest reliability of the Hearing Handicap Inventory for the Elderly. Ear Hear. 1986;7(5):295-99. [PMID: 3770324 DOI:10.1097/00003446-198610000-00002

15. Gorsuch RL. Factor analysis. 2nd ed. Hillsdale (NJ): Lawrence Erlbaum; 2003.

16. Humes LE. Modeling and predicting hearing aid outcome. Trends Amplif. 2003;7(2):41-75. [PMID: 15004647] DOI:10.1177/108471380300700202

17. Cohen J. Statistical power analysis for the behavioral sciences. 2nd ed. Hillsdale (NJ): Lawrence Erlbaum; 1988.

Submitted for publication April 30, 2010. Accepted in revised form January 24, 2011.

This article and any supplementary material should be cited as follows:

Johnson EE. Determinants of Department of Veterans Affairs hearing aid brand dispensing by individual audiologists. J Rehabil Res Dev. 2011;48(7):865-80.

DOI:10.1682/JRRD.2010.04.0077

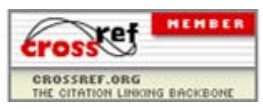

